\title{
Prospecciones electromagnéticas en el yacimiento arqueológico de Japoto, Manabí, Ecuador
}

Prospections électromagnétiques sur le site archéologique de Japoto, Manabí, Équateur

Electromagnetic survey at the Japoto archaeological site, Manabí, Ecuador

Carlos Mayo, Mercedes Guinea y Julia Mayo

\section{(2) OpenEdition}

Journals

\section{Edición electrónica}

URL: http://journals.openedition.org/bifea/1765

DOI: 10.4000/bifea.1765

ISSN: 2076-5827

\section{Editor}

Institut Français d'Études Andines

\section{Edición impresa}

Fecha de publicación: 1 diciembre 2010

Paginación: 577-587

ISSN: 0303-7495

\section{Referencia electrónica}

Carlos Mayo, Mercedes Guinea y Julia Mayo, « Prospecciones electromagnéticas en el yacimiento arqueológico de Japoto, Manabí, Ecuador », Bulletin de l'Institut français d'études andines [En línea], 39 (3) | 2010, Publicado el 01 junio 2011, consultado el 08 diciembre 2020. URL : http:// journals.openedition.org/bifea/1765; DOI : https://doi.org/10.4000/bifea.1765

\section{(ब) $\Theta \Theta$}

Les contenus du Bulletin de l'Institut français d'études andines sont mis à disposition selon les termes de la licence Creative Commons Attribution - Pas d'Utilisation Commerciale - Pas de Modification 4.0 International. 


\title{
Prospecciones electromagnéticas en el yacimiento arqueológico de Japoto, Manabí, Ecuador
}

\author{
Carlos Mayo* \\ Mercedes Guinea** \\ Julia Mayo**
}

\section{Resumen}

En el año 2007 se encontró en el yacimiento arqueológico de Japoto, provincia de Manabí, Ecuador, una estructura prehispánica de adobe y quincha bajo uno de los montículos del sitio. Dos años más tarde, una vez realizada la excavación parcial de dicha estructura, Carlos Mayo realizó una prospección electromagnética en este yacimiento con el objeto de establecer, en lo posible, los límites de la estructura en la parte no excavada y determinar si ésta forma parte de un edificio aislado o de un complejo arquitectónico de mayor envergadura. Los resultados del trabajo de campo nos permitieron lanzar la hipótesis de que efectivamente el edificio estuvo formando parte de un complejo mayor.

Palabras clave: prospección geofísica, conductividad, estructuras arquitectónicas

* Científico Asistente del Instituto Tropical de Investigaciones Tropicales. Candidato a Doctor por la Universidad de Santiago de Compostela. Dirección: Smithsonian (STRI). APDO 0843-03092. Ancón, Panamá Ciudad Capital. E-Mail: mayoc@si.edu

** Profesora Titular Universidad Complutense de Madrid. Departamento de Historia de América II. Facultad de Geografía e Historia. C/ Profesor Arangure s/n, 28040 Madrid, España. E-Mail: guinea@a ghis.ucm.es

${ }^{* * *}$ Científica Asociada del Instituto Tropical de Investigaciones Tropicales. Dirección: Smithsonian (STRI). APDO 0843-03092. Ancón, Panamá Ciudad Capital. E-Mail: mayoj@si.edu 


\title{
Prospections électromagnétiques sur le site archéologique de Japoto, Manabí, Équateur
}

\section{Résumé}

En 2007, une structure préhispanique d'adobe et de quincha a été découverte sous l'un des monticules du complexe archéologique de Japoto, province de Manabí, Équateur. Deux ans plus tard, après avoir réalisé une fouille partielle de cette structure, Carlos Mayo a effectué une prospection électromagnétique sur le site afin d'établir, dans la mesure du possible, les limites de la structure sur la zone non fouillée, et de déterminer si elle faisait partie d'un édifice isolé ou d'un complexe architectural de plus grande envergure. Les résultats de ce travail de terrain permettent d'émettre I'hypothèse que cet édifice appartenait effectivement à un complexe plus grand.

Mots clés : prospection géophysique, conductivité, structure architectonique, Manabí

\section{Electromagnetic survey at the Japoto archaeological site, Manabí, Ecuador}

\begin{abstract}
In 2007, a Prehispanic structure made of adobe and quincha was found the archaeological site of Japoto, in the Manabí province, Ecuador. The structure was under one of the mounds at the site, a portion of the structure was excavated. In 2009, Carlos Mayo made an electromagnetic survey there in order to establish the limit of the structure. The goal was to understand whether the structure was an isolated building or part of a larger architecture complex. The investigation suggested that the structure was part of a large architectural compound.
\end{abstract}

Key words: Geophysical survey, conductivity, architectural structures, Manabi

\section{INTRODUCCIÓN}

Desde los años 1960 las prospecciones geofísicas vienen siendo aplicadas con éxito en el campo de la arqueología en Europa (Tite \& Mullisn, 1970) y en los Estados Unidos (Bevan, 1983). En hispanoamérica se han realizado más tarde y con distintas técnicas tanto en yacimientos precolombinos (Mojica et al., 2007; Sebastian et al., 2005) como coloniales (Chávez et al., 2005; Mojica et al., 2006), demostrando ampliamente su utilidad a la hora de su identificación e interpretación.

En la primera semana de julio de 2009, los autores han realizado prospecciones geofísicas en el yacimiento arqueológico de Japoto, dentro de los trabajos de campo del Proyecto Manabí Central que desde 2004 dirige Jean-François Bouchard y cuyos resultados preliminares son el objeto de este artículo. Este yacimiento perteneciente a la cultura manteña, se encuentra localizado a $28 \mathrm{~km}$ de Bahía de Caraquez y a $2 \mathrm{~km}$ hacia el interior de la población costera de San Jacinto en Manabí. El yacimiento se presenta en el terreno como una agrupación de montículos de diversos tamaños, visibles en las zonas clareadas de vegetación (ver Bouchard en este mismo volumen). Durante los tres primeros años de 
excavaciones, los trabajos han revelado que dichos montículos son el resultado de una combinación de desechos habitacionales y elevaciones artificiales del terreno (Guinea, 2006a; 2006b; Touchard, 2006), con la excepción de uno que tiene un carácter funerario (Delabarde, 2006). Sin embargo, en 2007, el equipo de la Universidad Complutense perteneciente al proyecto citado localiza una estructura arquitectónica de tierra (ver Guinea en este mismo volumen) dentro de uno de los montículos. Comprobar si ésta es una estructura aislada o forma parte de un complejo arquitectónico de mayores dimensiones ha sido de vital importancia para la interpretación del significado de dicha estructura y del yacimiento en su conjunto, así como para la valoración de las acciones a tomar en el futuro con respecto a la intervención del sitio. Con este objetivo principal, y otros de carácter más puntual como definir el límite oeste del edificio localizado, se han realizado los trabajos de prospección geofísica que se presentan a continuación.

\section{TÉCNICA DE PROSPECCIÓN GEOFÍSICA APLICADA: CONDUCTIVIDAD ELECTROMAGNÉTICA}

Existen diversos métodos para la medición de las propiedades físicas de los suelos. Los más utilizados en prospecciones arqueológicas son la susceptibilidad magnética, la resistividad eléctrica y la conductividad electromagnética. Esta última ha sido la elegida para la prospección geofísica en Japoto, concretamente con el método Slingram de conductividad electromagnética, empleando un equipo EM 38 (Geonics Limited 2001) en modo vertical capaz de medir de manera precisa y rápida la conductividad eléctromagnética del subsuelo expresada en valores de milisiemens por metro. Esta medida es proporcionalmente inversa a la resistividad eléctrica. La conductividad es medida a través de corrientes eléctricas que son inducidas por un campo magnético a través de dos bobinas. Estas corrientes crean un campo magnético secundario que es medido por la bobina receptora. La separación entre las bobinas es proporcional a la profundidad de las mediciones. En el caso de EM 38 la separación es de un metro por lo que es capaz de medir, en modo vertical, las propiedades físicas del terreno, con una profundidad de hasta un metro y medio.

En general los valores de conductividad obtenidos son producto de la composición y formación natural de los suelos y dependen de las porciones relativas de arenas, limo y arcilla presentes. Pero en ocasiones se pueden detectar cambios en cuanto a la composición, la textura, las propiedades químicas y mineralógicas que pueden relacionarse con actividades.

Para la interpretación de los resultados de un trabajo de este tipo es muy necesario tener en consideración el tipo de suelos en el que se van a efectuar. El yacimiento de Japoto está situado en una llanura de litoral caracterizada por una vegetación densa de bosque tropical seco. La composición de los suelos en esta región se ve afectada por la erosión marina de los acantilados (Usselmann, 2006; Dumont et al., 2006) y por la sedimentación arcillosa originada por las precipitaciones cíclicas 
durante los fenómenos de El Niño. Como podemos observar en el mapa de suelos del yacimiento (fig. 1), al norte dominan materiales procedentes de escorrentía y colusiones mientras que en la zona donde se realizaron las prospecciones geofísicas predominan los materiales sedimentarios producto del desbordamiento de los esteros de curso estacional.

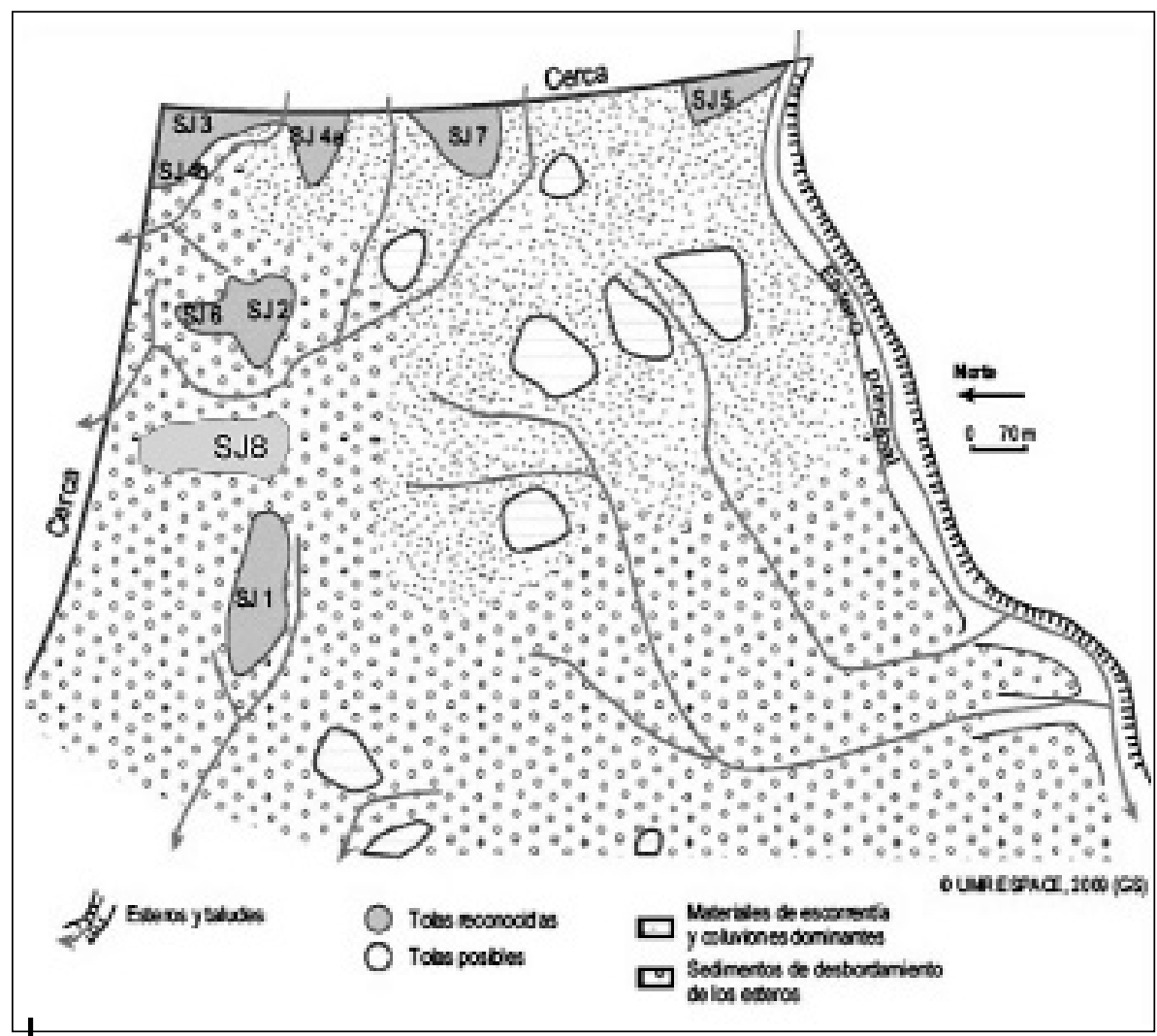

Figura 1 - Suelos de yacimiento arqueológico de Japoto

Modificado a partir de Usselmann (2006: fig. 6)

\section{METODOLOGÍA}

Tras un reconocimiento preliminar del yacimiento arqueológico, decidimos realizar prospecciones electromagnéticas en modo vertical sobre los montículos $\mathrm{J1}$ y J8 (fig. 2), así como las zonas adyacentes al oeste, sur y al este de este último con el fin de cubrir los objetivos propuestos: fijar los límites de la edificación hallada en el montículo J8 y determinar si es una estructura aislada o forma parte de un conjunto arquitectónico mayor.

Para ello se trazaron 12 polígonos de prospección (fig. 3) en los cuales se proyectaron una serie de transectos longitudinales con una separación de un 


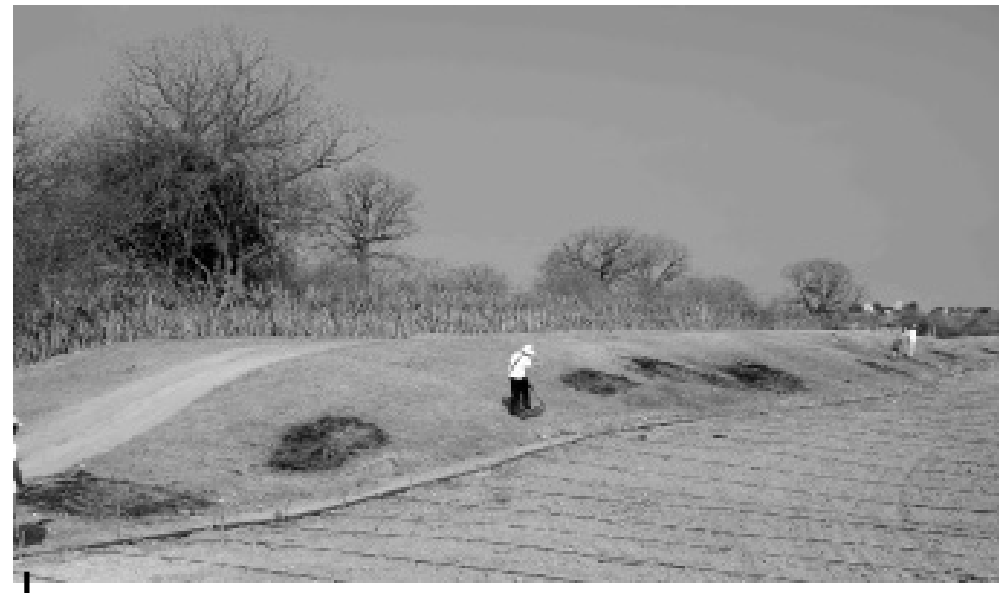

Figura 2 - Carlos Mayo realizando labores de prospección geofísica sobre la superficie del montículo J1

Foto: M. Guinea

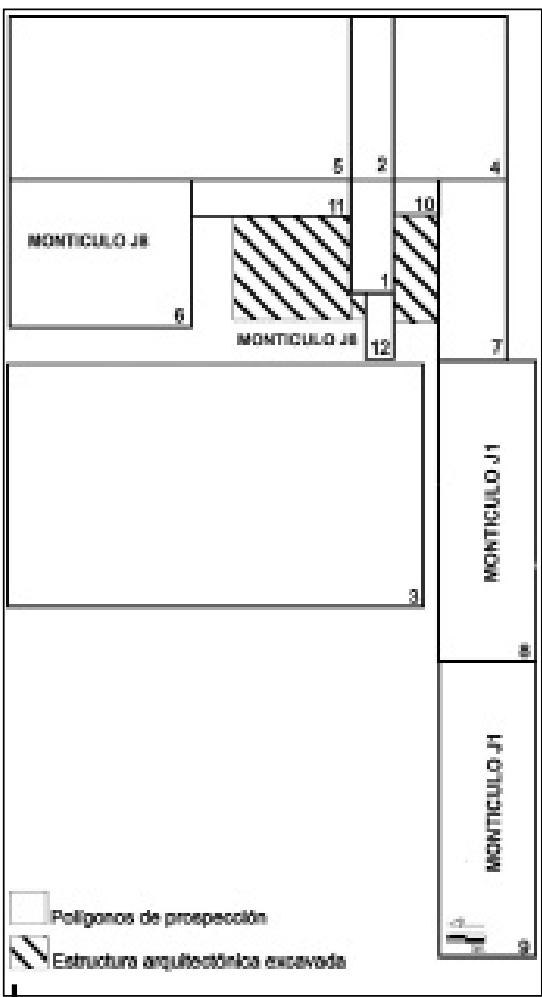

Figura 3 - Situación de los polígonos de prospección geofísica en relación a la estructura E1 del J8

Dibujo: C. Mayo metro. Se hicieron recorridos sobre ellos recogiendo y almacenando valores cada metro. Estos datos fueron volcados con posterioridad a una base de datos que nos permitió generar planimetrías a través del programa informático Surfer Golden 8.

\section{RESULTADOS DE LA PROSPECCIÓN}

Los valores de conductividad del área prospectada oscilan entre mínimos de $26 \mathrm{~ms} / \mathrm{m}$ y máximos de 127 $\mathrm{ms} / \mathrm{m}$. Los valores de baja conductividad corresponden a la zona no excavada en el cuerpo sur del montículo J8, un área adyacente al SE del mismo, la totalidad del montículo J1 y un canal de escorrentía que separa ambos. Los valores de alta conductividad se recogieron sobre el cuerpo norte del montículo J8 y las zonas contiguas al E y W (fig. 4). Más detalladamente, en la planimetrías generadas se observan cinco anomalías electromagnéticas (fig. 5):

\section{1. Anomalía 1}

Esta anomalía mide aproximadamente 16 metros de longitud, tiene una orientación E-W y se encuentra en el polígono de prospección 2 (PP2), donde los valores 


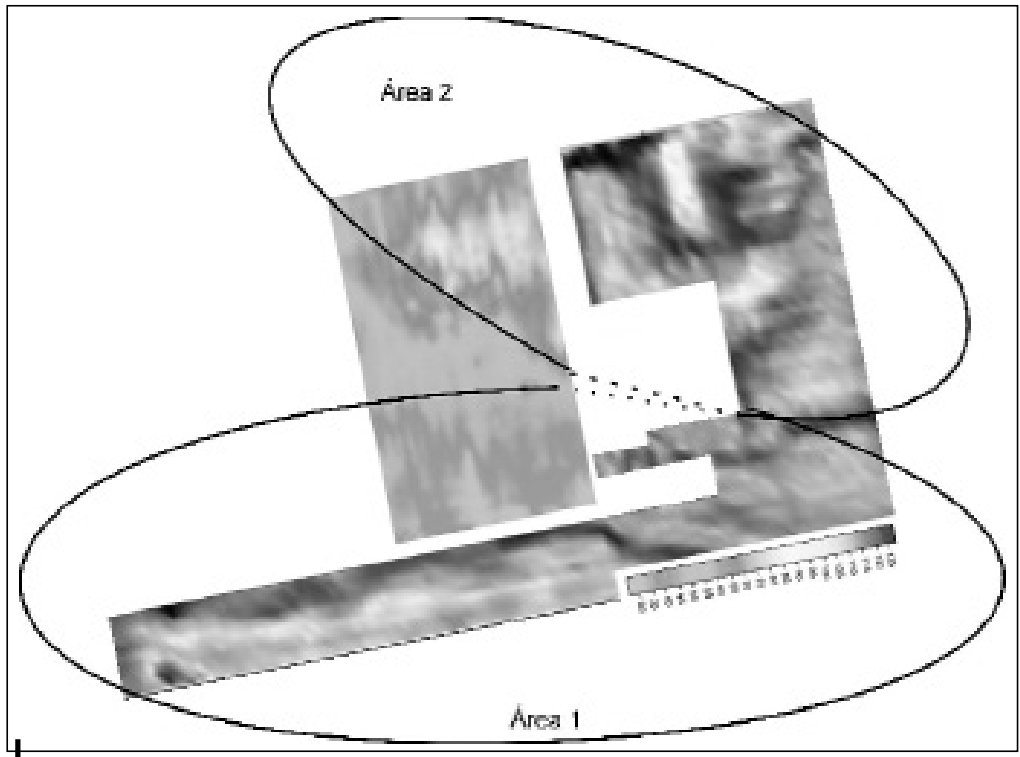

Figura 4 - Mosaico de las planimetrías geofísicas con la delimitación de los distintos valores de conductividad

Bajos Área 1, altos Área 2: C. Mayo

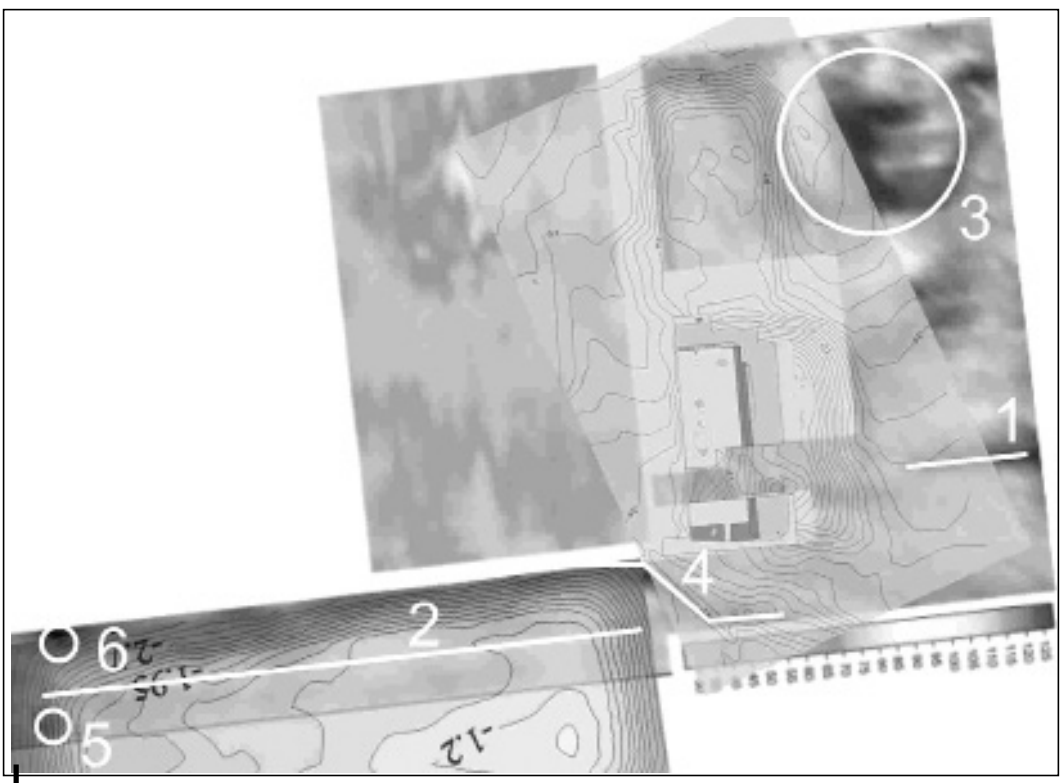

Figura 5 - Mosaico de las planimetrías geofísicas con la situación de la E1, la superposición de la topografía de los montículos J8 y J1 y la localización de las distintas anomalías referidas en el texto

C. Mayo 
de alta y baja conductividad se agrupan en dos zonas con límites bien definidos en cuyos límites se proyecta una geometría lineal (figs. 5 y 6). Es importante señalar que esta geometría, que responde, como hemos señalado, a cambios en los valores de conductividad, no puede ser en ningún caso producida por la topografía del yacimiento.

\section{2. Anomalía 2}

En el polígono de prospección 8 (PP8) situado sobre el montículo J1, esta geometría está originada por un ligero incremento en los valores de conductividad a lo largo de un eje Este/Oeste (misma orientación que la Anomalía 1), en una zona homogénea de baja conductividad (figs. 5 y 7). Esta es la anomalía más significativa de todas las observadas hasta el momento. Creemos que se trata de una anomalía de origen

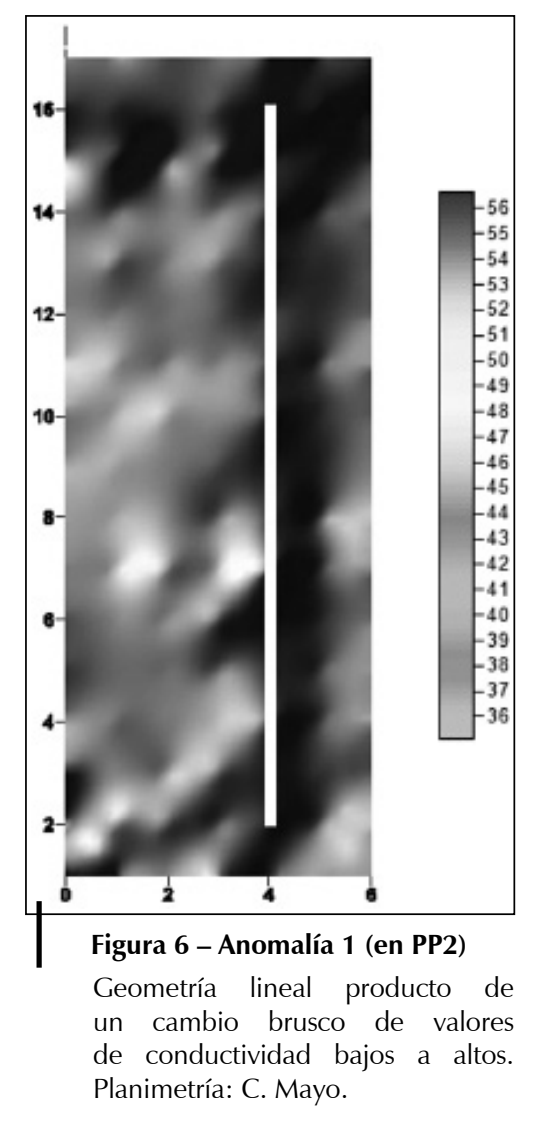

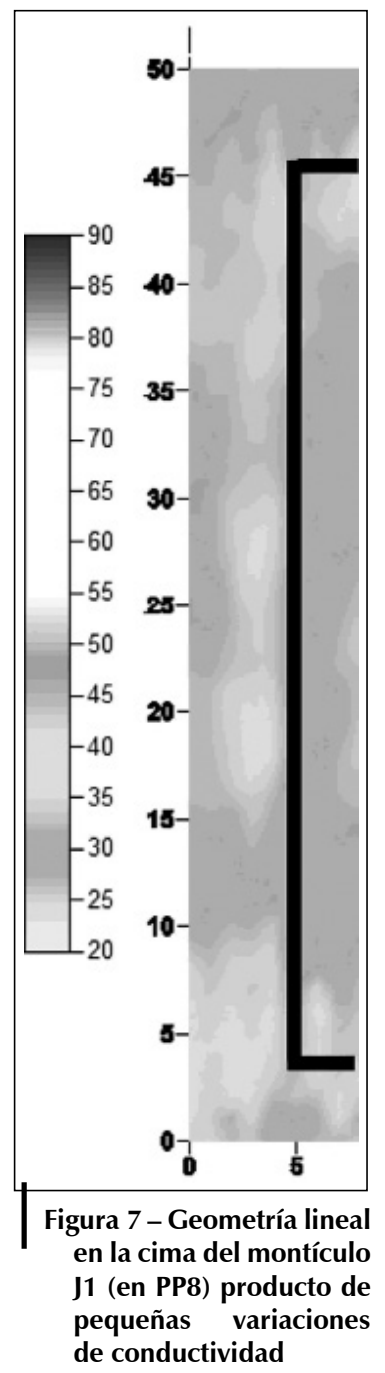

Planimetría: C. Mayo 
antropogénico dadas sus dimensiones y su orientación perpendicular al eje de la estructura enterrada bajo el montículo J8, lo que podría indicar que existe una relación espacial-estructural de conjunto entre ambas.

\section{3. Anomalía 3}

En los polígonos de prospección 5 (PP5) y 6 (PP6) se observaron valores de conductividad de altos a muy altos formando una geometría circular (figs. 5 y 8). Creemos que se trata de un área deprimida en la que se ha depositado material sedimentario. Se trata a nuestro entender de anomalías naturales, no antropogénicas.

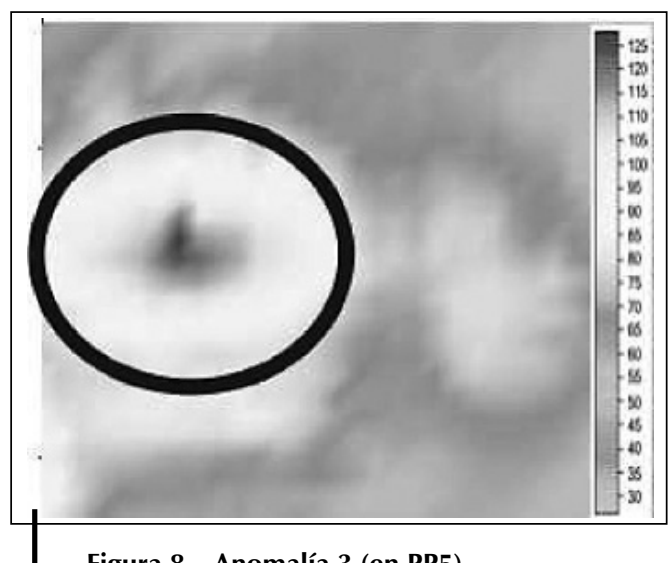

Figura 8 - Anomalía 3 (en PP5)

Rasgo circular producto de una oscilación gradual de valores de conductividad bajos a altos

\section{4. Anomalía 4}

Esta es una geometría en zig-zag que corresponde a una quebrada estacional (canal) visible en superficie y que se detectó en los polígonos de prospección 7 y 8 (PP7 y PP8) (fig. 5). Es posible que, al menos en parte, esta geometría se deba a un efecto natural de topografía.

\section{5. Anomalías 5 y 6}

Estas son anomalías puntuales y muy fuertes localizadas en el polígono de prospección 9 (PP9) (fig. 5). Son dos casos en los que se producen cambios bruscos en los registros de conductividad sobre un único punto de prospección dentro de un área de valores de conductividad homogéneos (fig. 9). Su origen es indeterminado, posiblemente antropogénico.

Al margen de las geometrías detectadas, hemos observado que existen valores similares de conductividad entre los montículos J1 y el cuerpo sur del J8, los cuales son totalmente distintos a los recogidos en el cuerpo norte de este último montículo (fig. 5). El hecho de que los valores de conductividad sean tan diferentes dentro incluso de un mismo montículo nos indica que es posible detectar cuando estos contienen elementos estructurales o rellenos diferentes.

\section{CONCLUSIONES}

Como hemos dicho más arriba, los objetivos principales de las prospecciones geofísicas del sitio de Japoto, han sido buscar otras posibles estructuras arquitectónicas en las inmediaciones de la E1 del J8 y hallar el límite oeste del 


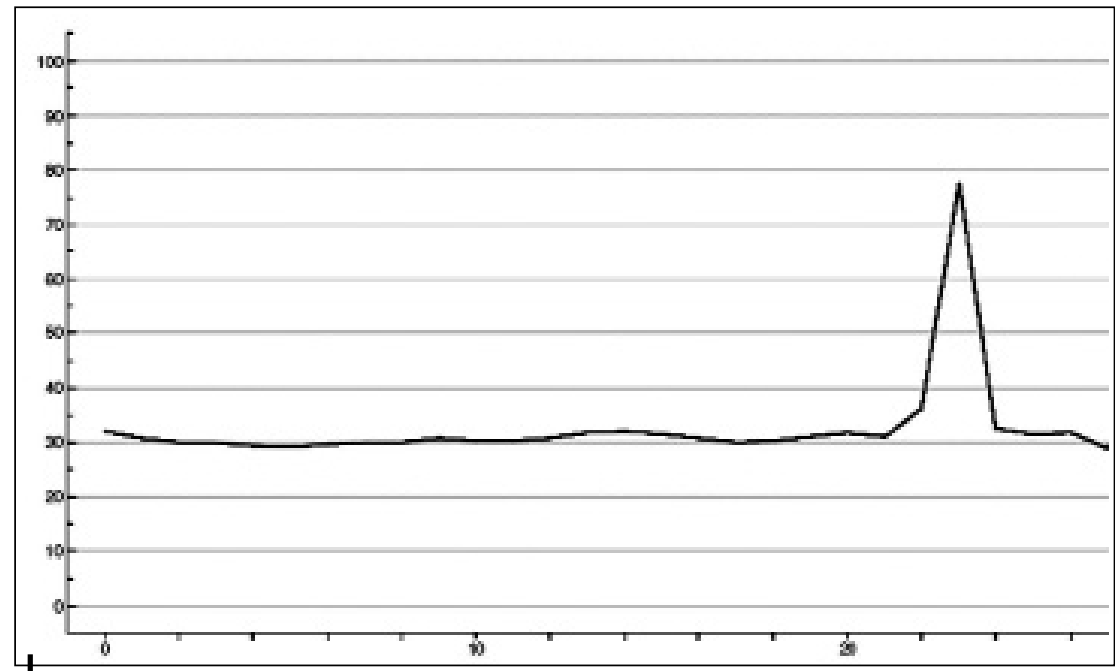

Figura 9 - Transecto de prospección en el montículo J1 en el que se observa un cambio brusco en la conductividad sobre la Estación 24 dentro de un área con una conductividad con valores homogéneos

edificio. Lo primero que salta a la vista, al observar el mosaico de planimetrías geofísicas, es que existen dos áreas bien definidas con valores de conductividad diferentes (fig. 4). El Área 1, prácticamente la mitad sur del área prospectada, presenta valores de conductividad bajos de forma relativamente homogénea. Interpretamos esta zona como un «área construida» o antropogénica, ya que los valores en toda ella son semejantes a los se recogen en la zona del testigo no excavado del montículo J8 (fig. 5), donde se sabe, por los sondeos realizados, que se encuentra parte de la estructura arquitectónica (E1) excavada. Se destaca que, al sureste y suroeste de dicha estructura, aparecen lo que pueden ser los límites de otras dos (fig. 5: líneas 1 y 2). Por el contrario, el Área 2 (fig. 4), en la mitad norte y con valores de conductividad altos, parece ser una zona de rellenos y geometrías naturales.

En cuanto a las anomalías, las planimetrías muestran seis geometrías. Estamos proponiendo un origen natural para dos de ellas, una circular (figs. 8 y $5 \mathrm{n} .^{\circ}$ ) y otra en zig-zag (fig. 5 n. ${ }^{\circ}$ ), dado que corresponden a dos elementos geomorfológicos observables en superficie, una quebrada y una depresión sedimentaria, y un origen humano para las otras cuatro, dos puntuales (fig. 5 n. 5 y 6 ) y dos lineales (figs. 6,7 y $5 \mathrm{n} .^{\circ} 1$ y 2). En el caso de la anomalía circular (figs. 8 y $5 \mathrm{n} .^{\circ} 3$ ), no hay que descartar la posibilidad de que esta depresión sea el resultado de una excavación humana antigua, aunque no hemos descartado la hipótesis de un origen natural como la más probable. Sabemos que en la zona se han construido montículos usando rellenos y aunque parte de estos provengan de zonas algo distantes (ver Guinea en este mismo volumen), no hay que descartar que otra parte fuera extraída de zonas más inmediatas. Tenemos experiencia (Mayo et al., 2007) en otros paisajes culturales en los que las excavaciones de canales o 
socavones han sido una actividad complementaria a la construcción con rellenos. De ser este el caso, cabría esperar que parte de los rellenos de algunos de los montículos proviniesen de este «socavón» o depresión del que estamos hablando, siendo necesario, para la confirmación de esta hipótesis alternativa, extraer muestras de su contenido y compararlas con las variedades de rellenos de los montículos ya excavados. Las anomalías antropogénicas son lógicamente las de mayor interés y se encuentran situadas en torno al edificio excavado (E1) bajo el montículo 38 y debajo de del montículo J1 (fig. 5 n. ${ }^{\circ}$ 1, 2, 6 y 7), pareciendo estar dispuestas de manera ordenada, por lo que podrían interpretarse como segmentos arquitectónicos de otros edificios los cuales formarían, junto al ya conocido, un conjunto de mayores dimensiones.

En relación al segundo objetivo, la localización del límite oeste de la estructura (E1) excavada en 2007 y 2008, necesitamos tomar más mediciones para poderlo determinar con precision. No obstante, los valores de las mediciones tomadas en el límite $W$ del testigo del área excavada (fig. 5) muestran un cambio sutil a lo largo de un eje N/S paralelo al de la estructura, alcanzando valores entre los 60 a $70 \mathrm{~ms} / \mathrm{m}$. Eso hace posible que esté en relación con el buscado talud W de la plataforma sobre la que se levanta la estructura. Siguiendo hacia el oeste, se obtienen unos valores de conductividad intermedios, entre los 30 y los 50 $\mathrm{ms} / \mathrm{m}$, que pueden ser a su vez interpretables como un espacio, sí no construido, sí alterado (vertido de carbones, áreas quemadas, etc.) que pudo corresponder a una plaza escenario de diversas actividades, aunque insistimos en que, como hemos dicho más arriba, todavía faltan datos para fundamentar estas hipótesis.

Finalmente, se establece la clara diferencia en los valores de conductividad entre los cuerpos norte y sur del montículo J8 (figs. 4 y 5). Los valores del primero de ellos, mucho más altos que los del segundo, donde se encuentra la estructura enterrada (E1), y los del montículo J1, nos están indicando la ausencia de restos arquitectónicos bajo este. Esto significa que los montículos visibles actualmente en el yacimiento no guardan una relación directa con la localización de otras posibles estructuras en su subsuelo y que la forma exterior compleja del montículo J8 no está determinada por la estructura que entierra en parte.

\section{Referencias citadas}

BEVAN, B., 1983 - Electromagnetics for Mapping Buried Earth Features. Journal of Field Archaeology, 10: 47-54.

CHÁVEZ, R. E., CÁMARA, M., PONCE, R. \& ARGOTE, D., 2005 - Use of geophysical methods in urban archaeological prospection: The Basilica de Nuestra Señora de La Salud, Patzcuaro, Mexico. Geoarchaeology, 20: 505-519.

DELABARDE, T., 2006 - Una secuencia de patrones funerarios manteños en la provincia de Manabí: primeros resultados de la tola J7, Sitio de Japotó. Bulletin de l'Institut Français d'Études Andines, 25 (3): 313-320. 
DUMONT, J.-F., SANTANA, E., VALDEZ, F., THAY, J.-P., USSELMANN, P., ITURRALDE, D. \& NAVARRETE, E., 2006 - Fan beheading and drainage diversion as evidence of a 3200-2800 BP earthquake event in the Esmeraldas-Tumaco seismic zone: A case study for the effects of great subduction earthquakes. Geomorphology, 74: 100-123.

GUINEA, M., 2006a - Un sistema de producción artesanal de cuentas de concha en un contexto doméstico manteño: Japoto (provincia de Manabí, Ecuador). Bulletin de I'Institut Français d'Études Andines, 25 (3): 299-313.

GUINEA, M., 2006b - El uso de tierras comestibles por los pueblos costeros del Periodo de Integración en los Andes septentrionales. Bulletin de I'Institut Français d'Études Andines, 25 (3): 321-335.

MAYO, J., MOJICA, A., RUIZ, A., MORENO, E., MAYO, C. \& DE GRACIA, I., 2007 Estructuras arquitectónicas incipientes y áreas de explotación minera prehispánica de las cuencas de los ríos Grande y Coclé del Sur, Panamá. Revista Española de Antropología Americana, 37 (1): 93-110. DOSSIER Gran Coclé: paisaje cultural del istmo de Panamá.

MOJICA, A., ACOSTA, L., GUÉRIN, R., HO, C. A. \& CABALlERO, O., 2006 - Geophysical Investigations in the Old Panama Archaeological Site. Revista Geológica de América Central, 33-34: 131-137.

MOJICA, A., MAYO, J., MAYO, C., CHANTADA, J. R., DE GRACIA, I. \& FLORSCH, N., 2007 - Resultados de las prospecciones magnética eléctrica del yacimiento arqueológico El Caño (NA-20), Gran Coclé, Panamá. Revista Española de Antropología Americana, 37 (1): 111-126.

SEBASTIAN, P., REYES, E. R., COSENZA, V., CHINCHILLA, O., MOJICA, A., PASTOR, L., VANNOESERLANDE, R. \& GUÉRIN, R., 2005 - Prospección geofísica en la zona de Cotzumalguapa: Resultados de la IV Escuela Centroamericana de Geofísica Aplicada. In: XVIII Simposio de Investigaciones Arqueológicas en Guatemala (J. P. Laporte, B. Arroyo \& H. Mejira, eds.): 968-976; Ciudad de Guatemala: Museo Nacional de Arqueologia y Etnologia.

TITE, M. S. \& MULLINS, C., 1970 - Electromagnetic Prospecting on Archaeological Sites Using a Soil Conductivity Meter. Archaeometry, 12 (1): 97-104.

TOUCHARD, A., 2006 - Una casa manteña puede esconder otra: evaluación preliminar de la tola J6 de Japoto, provincia de Manabí, Ecuador. Bulletin de I'Institut Français d'Études Andines, 25 (3): 285-298.

USSELMANN, P., 2006 - Dinámica geomorfológica y medio ambiente en los sitios arqueológicos Chirije y San Jacinto/Japoto (costa del Manabí central, Ecuador). Bulletin de l'Institut Français d'Études Andines, 35 (3): 257-264. 